\title{
Organically Capped Iridium Nanoparticles as High-Performance Bifunctional Electrocatalysts for Full Water Splitting in Both Acidic and Alkaline Media: Impacts of Metal-Ligand Interfacial Interactions
}

Yi Peng, ${ }^{a,+, \#}$ Qiming Liu, ${ }^{a, \dagger}$ Bingzhang Lu, ${ }^{a, \dagger}$ Ting He, ${ }^{a, b}$ Forrest Nichols, ${ }^{a}$ Xiao Hu, ${ }^{c}$ Tiffanie Huang, ${ }^{a}$ Grace Huang, ${ }^{a}$ Lizette Guzman, ${ }^{a}$ Yuan Ping, ${ }^{a, *}$ Shaowei Chen ${ }^{a, *}$

a Department of Chemistry and Biochemistry, University of California, 1156 High Street, Santa Cruz, CA, 95064, United States

b College of Chemistry and Chemical Engineering, Central South University, Changsha, Hunan 410083, China

c School of Environment and Energy, South China University of Technology, Guangzhou, Guangdong 510006, China

† These authors contributed equally to this work

\# Present address: Department of Chemistry, The University of Texas at Austin, 100 E 24th Street A5300, Austin, Texas 78712, USA

*E-mail: shaowei@ucsc.edu (S. Chen), yuanping@ucsc.edu (Y. Ping)

\section{List of Contents}

- 1 scheme

- 16 figures

- 3 tables

- 1 movie 

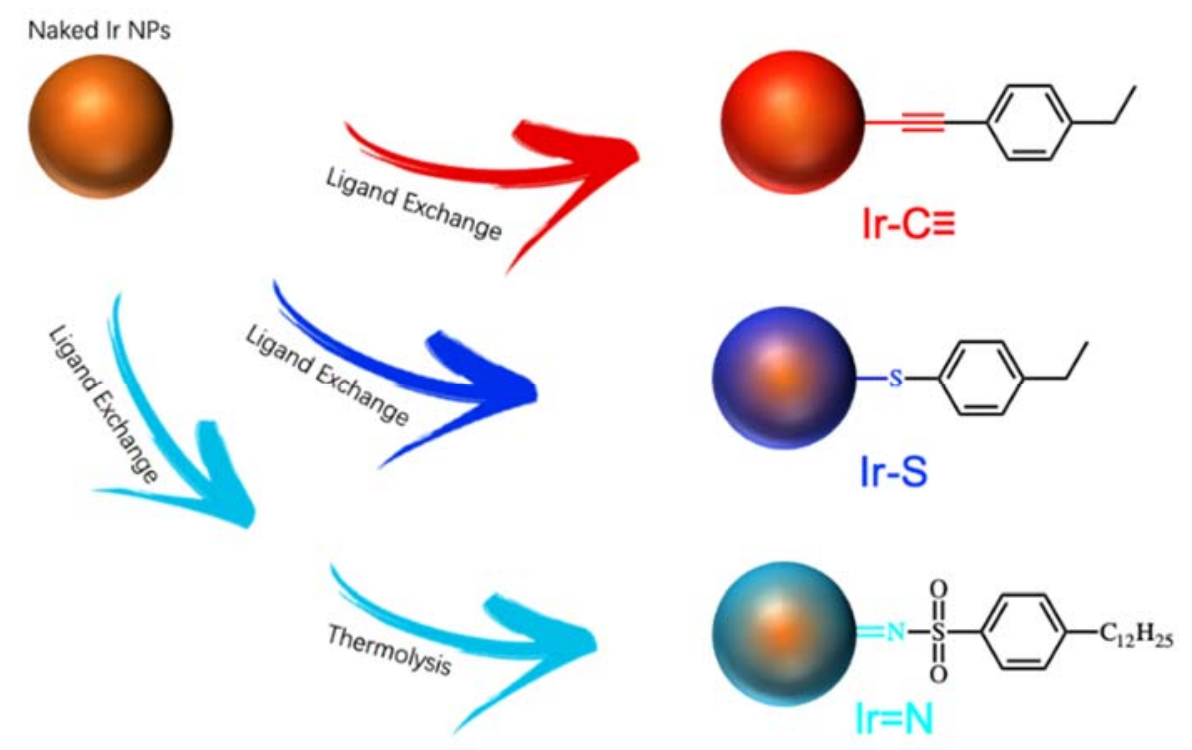

Scheme S1. Schematic illustration of the synthetic procedure of Ir nanoparticles capped with varied organic ligands. 

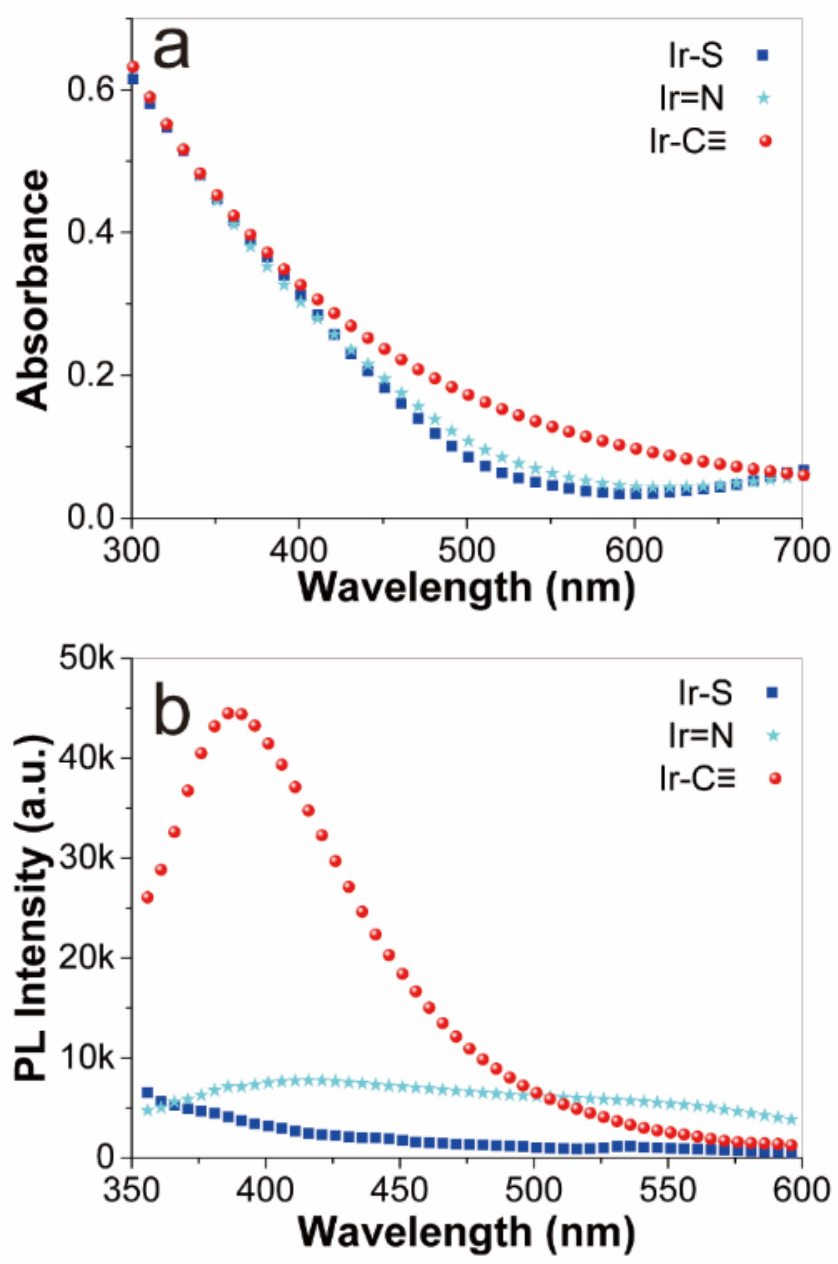

Figure S1. (a) UV-vis absorption and (b) photoluminescence emission spectra of the varied organically capped Ir nanoparticles. 


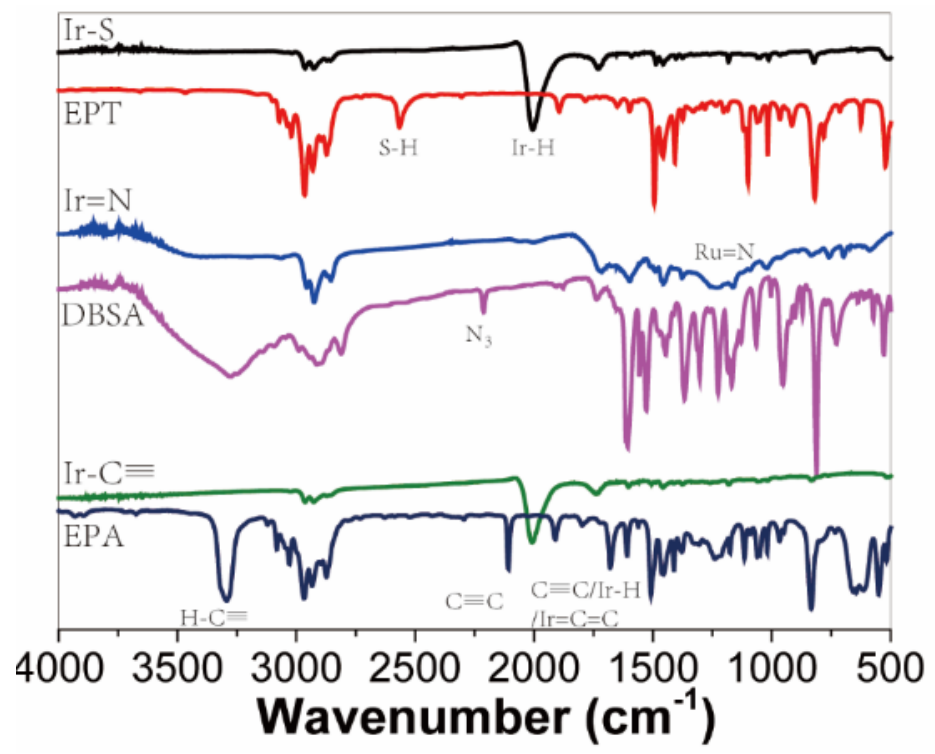

Figure S2. FTIR spectra of EPA, DBSA and EPT monomeric ligands and the corresponding organically capped Ir nanoparticles. 


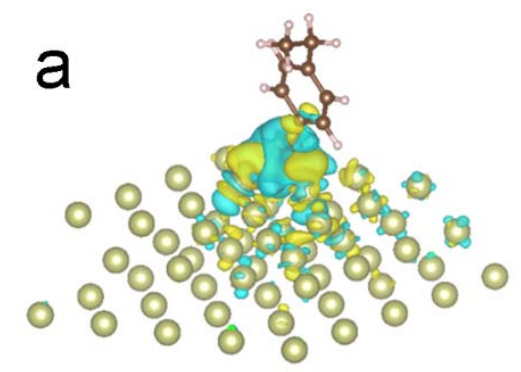

0.3878 e from slab to ligand

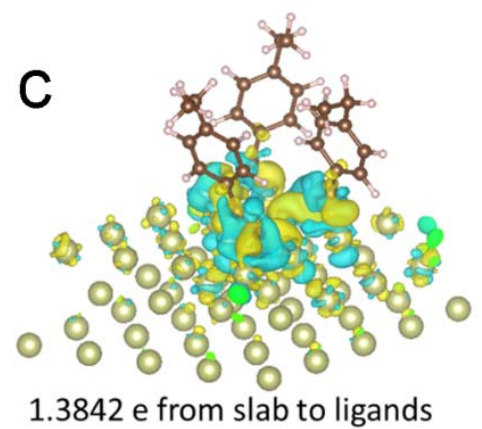

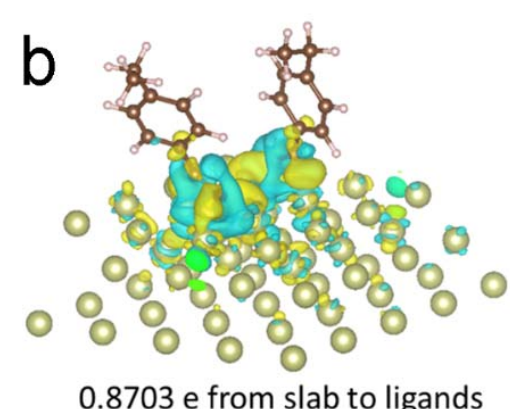

0.8703 e from slab to ligands

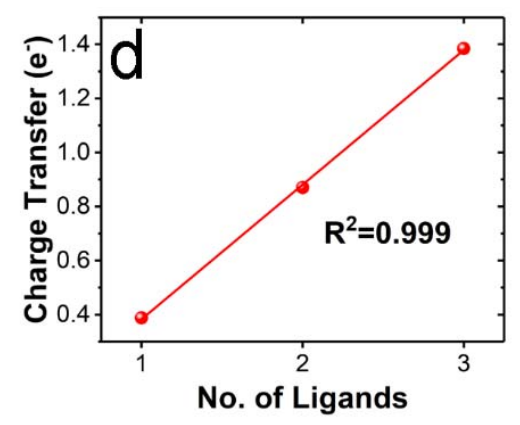

Figure S3. Optimized structures of $\operatorname{Ir}-\mathrm{C} \equiv$ with (a) one, (b) two, or (c) three ligands per slab and the corresponding interfacial charge density distribution. The cyan and yellow areas signify electron loss and electron gain, respectively. The isovalue of charge density is $0.001 \mathrm{e}^{-\mathrm{au}^{3}}$. (d) Linear correlation between charge transfer and the number of ligands on the slab.

The interfacial charge transfer is estimated to be 0.3878 e for one ligand, 0.8703 e for two ligands, and 1.3842 e for three ligands. That is, the interfacial charge transfer is linearly proportional to the number of surface capping ligands. 

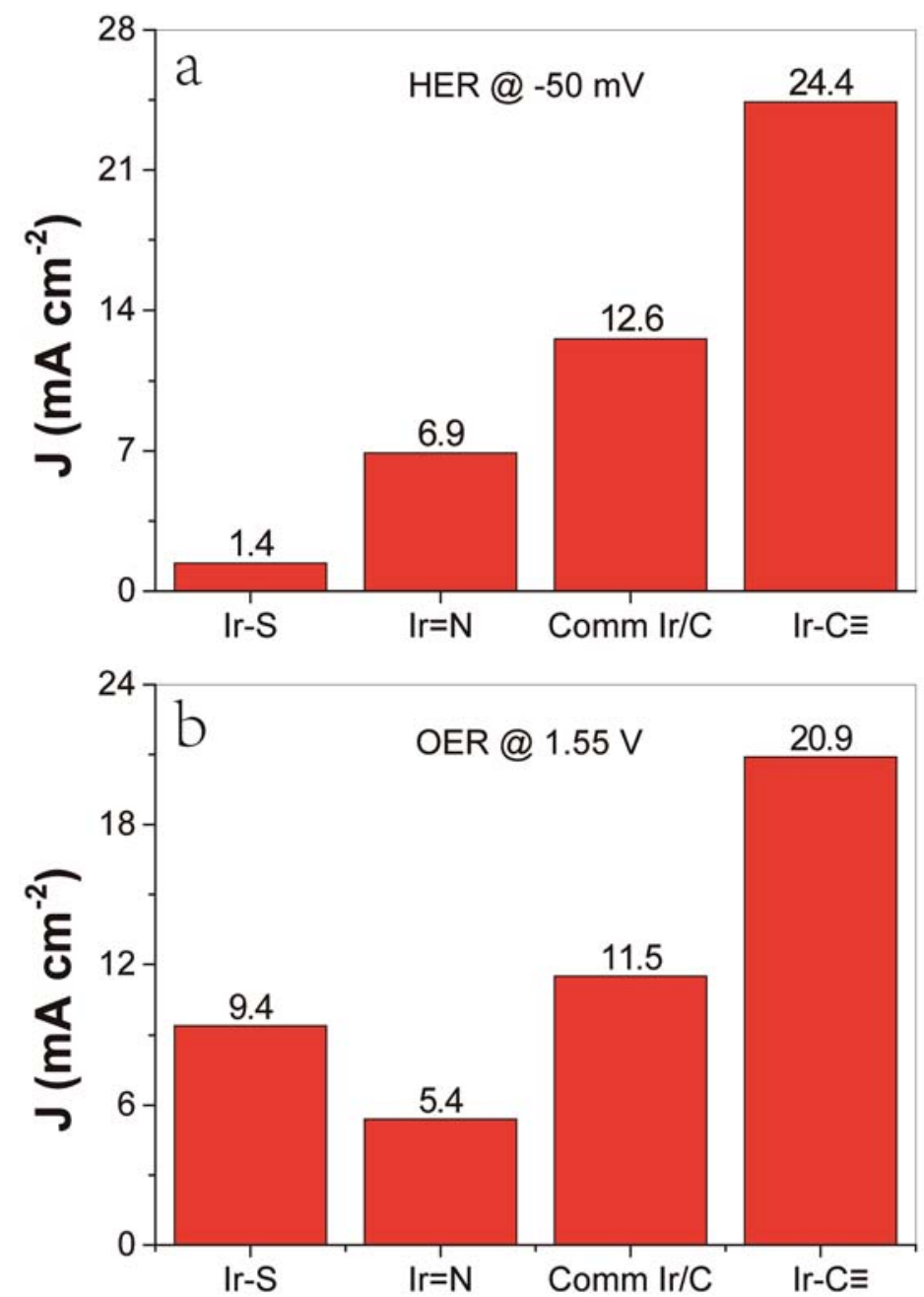

Figure S4. Comparison of the bifunctional catalytic performance in alkaline media. (a) HER current densities of the varied Ir nanoparticles at the potential of $-50 \mathrm{mV}$ in $1.0 \mathrm{M} \mathrm{KOH}$. (b) OER current densities of the varied Ir nanoparticles at the potential of $+1.55 \mathrm{~V}$ in $1.0 \mathrm{M} \mathrm{KOH}$. 

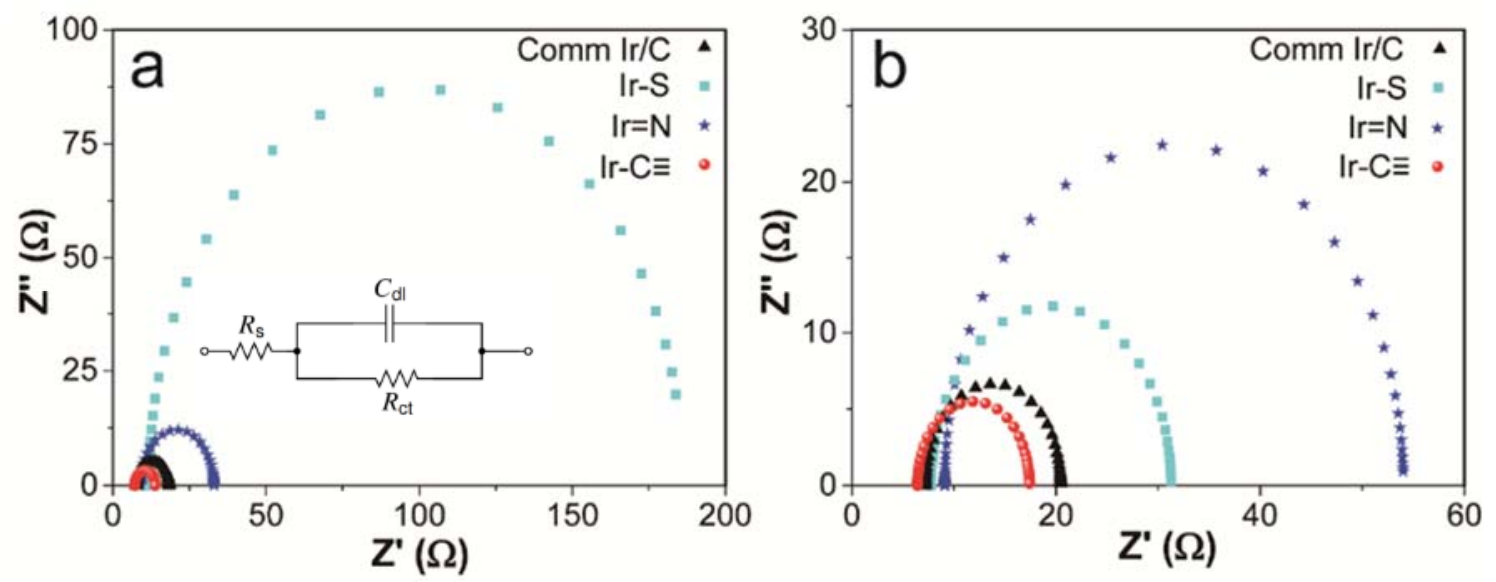

Figure S5. Nyquist plots at the potential of (a) $-50 \mathrm{mV}(\mathrm{HER})$ and (b) $1.55 \mathrm{~V}(\mathrm{OER})$ in $1 \mathrm{M} \mathrm{KOH}$. Inset is the Randle equivalent circuit: $R_{c t}$ is the charge transfer resistance, $C_{d l}$ is the double layer capacitance, and $R_{s}$ is the series resistance.

One can see that the $R_{s}$ increases in the order of $\mathrm{Ir}-\mathrm{C} \equiv<\mathrm{Ir}=\mathrm{N}<\mathrm{Ir}-\mathrm{S}$ (i.e., electrical conductivity increases in the reverse order), consistent with the variation of interfacial charge transfer from the Ir nanoparticle core to the capping ligands.

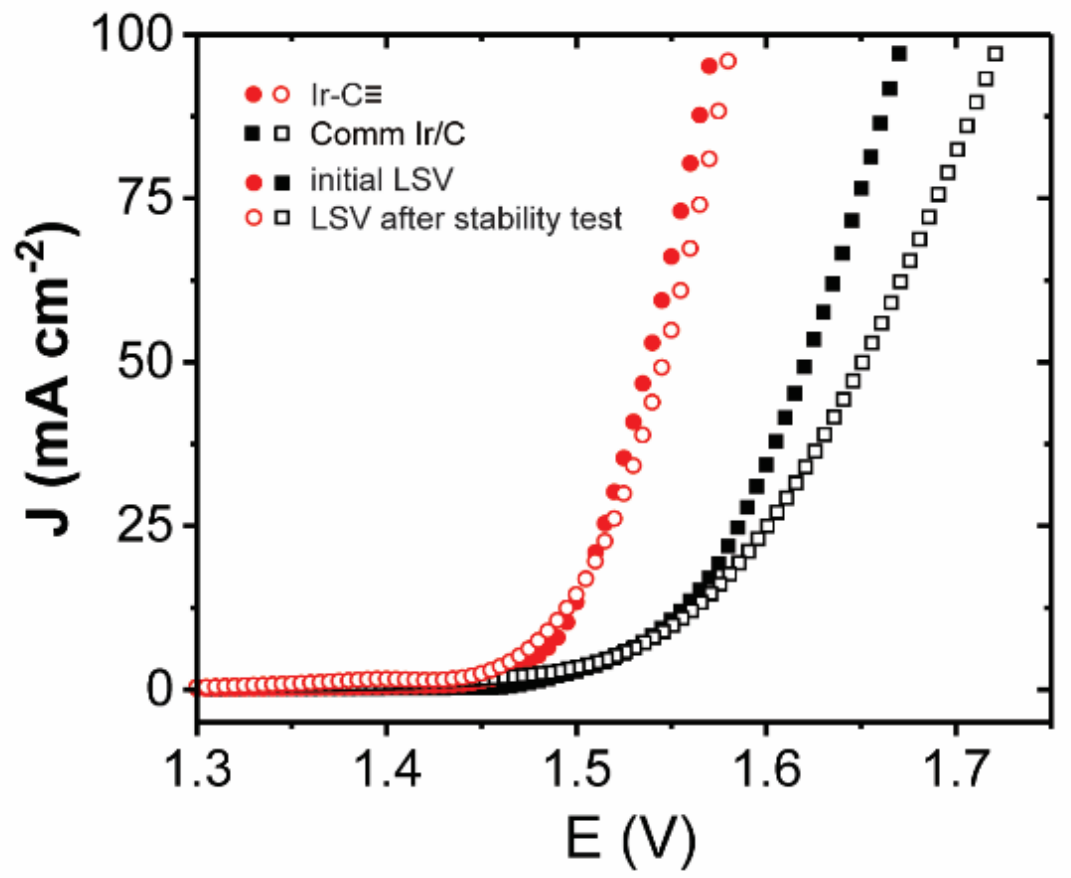

Figure S6. Current-voltage profiles of overall water splitting with $\mathrm{Ir}-\mathrm{C} \equiv$ and commercial $\mathrm{Ir} / \mathrm{C}$ as bifunctional electrocatalysts in $1.0 \mathrm{M} \mathrm{KOH}$ before and after the constant-potential stability test. 

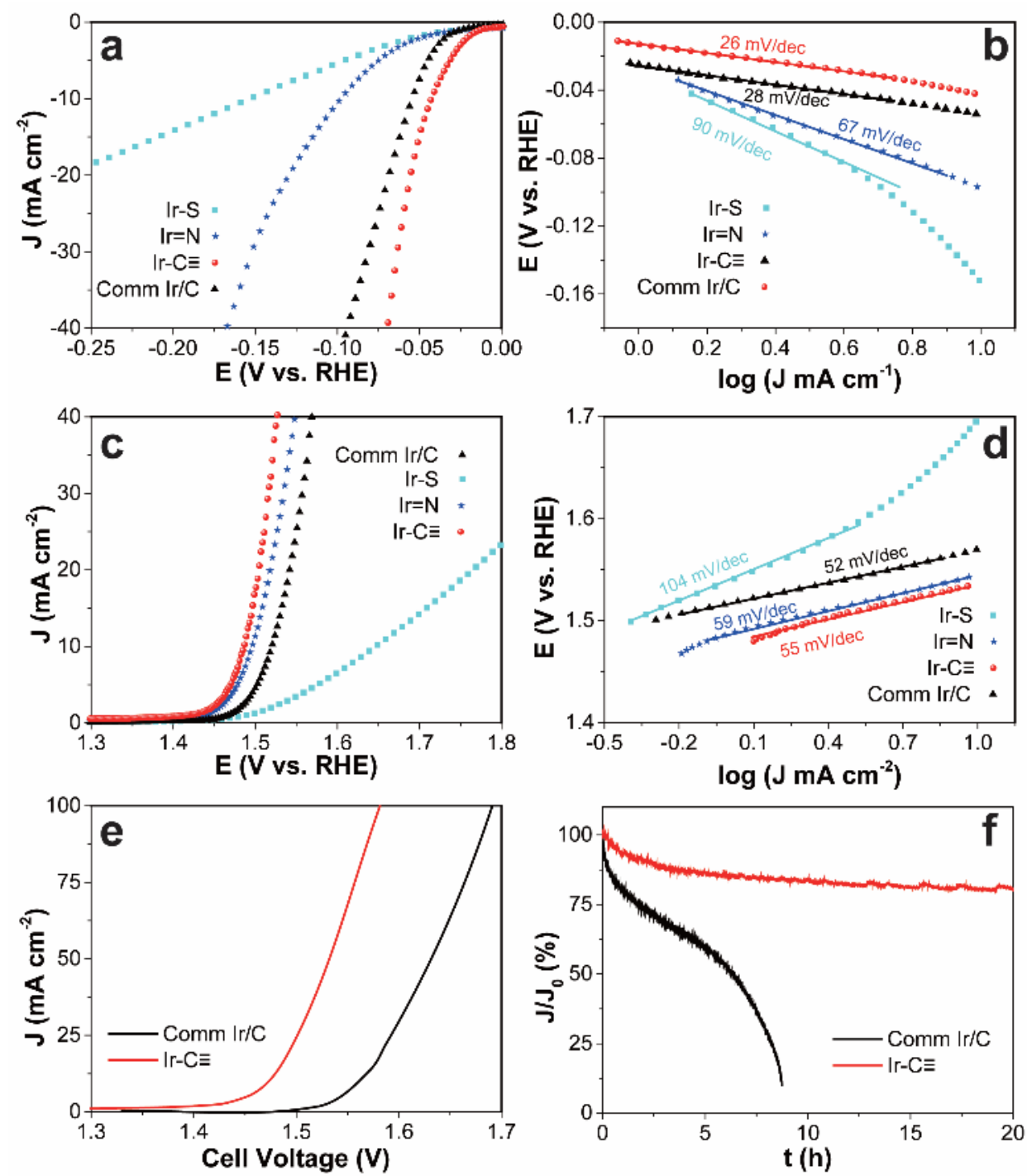

Figure S7. Bifunctional electrocatalytic performance towards HER and OER in $0.5 \mathrm{M} \mathrm{H}_{2} \mathrm{SO}_{4}$. (a) HER polarization curves of the series of electrocatalysts, and (b) the corresponding Tafel plots. (c) OER polarization curves of the series of electrocatalysts, and (d) the corresponding Tafel plots. (e) Current-voltage curves of overall water splitting using $\mathrm{Ir}-\mathrm{C} \equiv$ and commercial $\mathrm{Ir} / \mathrm{C}$ as the bifunctional catalysts in $0.5 \mathrm{M} \mathrm{H}_{2} \mathrm{SO}_{4}$, and (f) the corresponding activity retention test by chronoamperometric measurements at the respective initial $E_{10}$. 

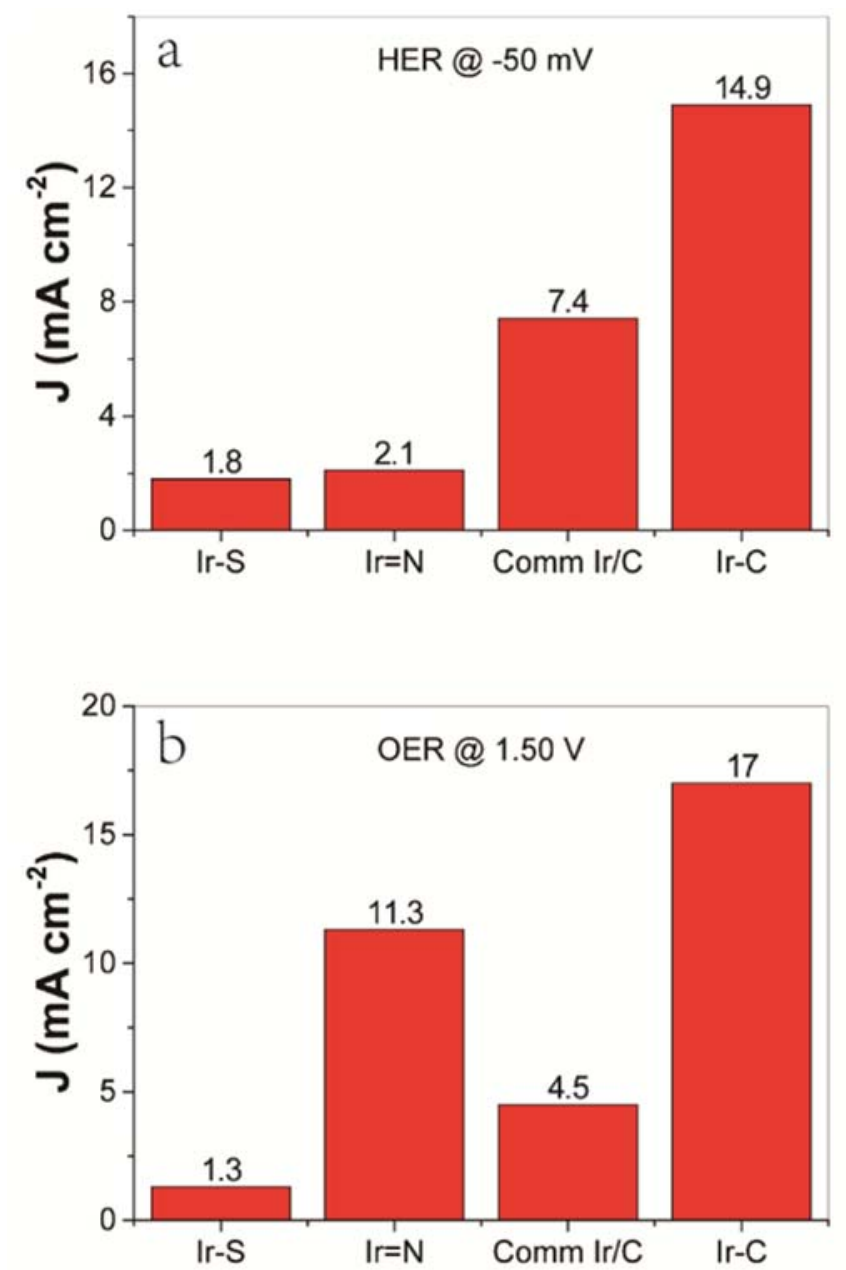

Figure S8. Comparison of the bifunctional catalytic performance in acidic media. (a) HER current densities of the various Ir nanoparticles at the potential of $-50 \mathrm{mV}$ in $0.5 \mathrm{M} \mathrm{H}_{2} \mathrm{SO}_{4}$. (b) OER current densities of the various Ir nanoparticles at the potential of $+1.55 \mathrm{~V}$ in $0.5 \mathrm{M} \mathrm{H}_{2} \mathrm{SO}_{4}$. 

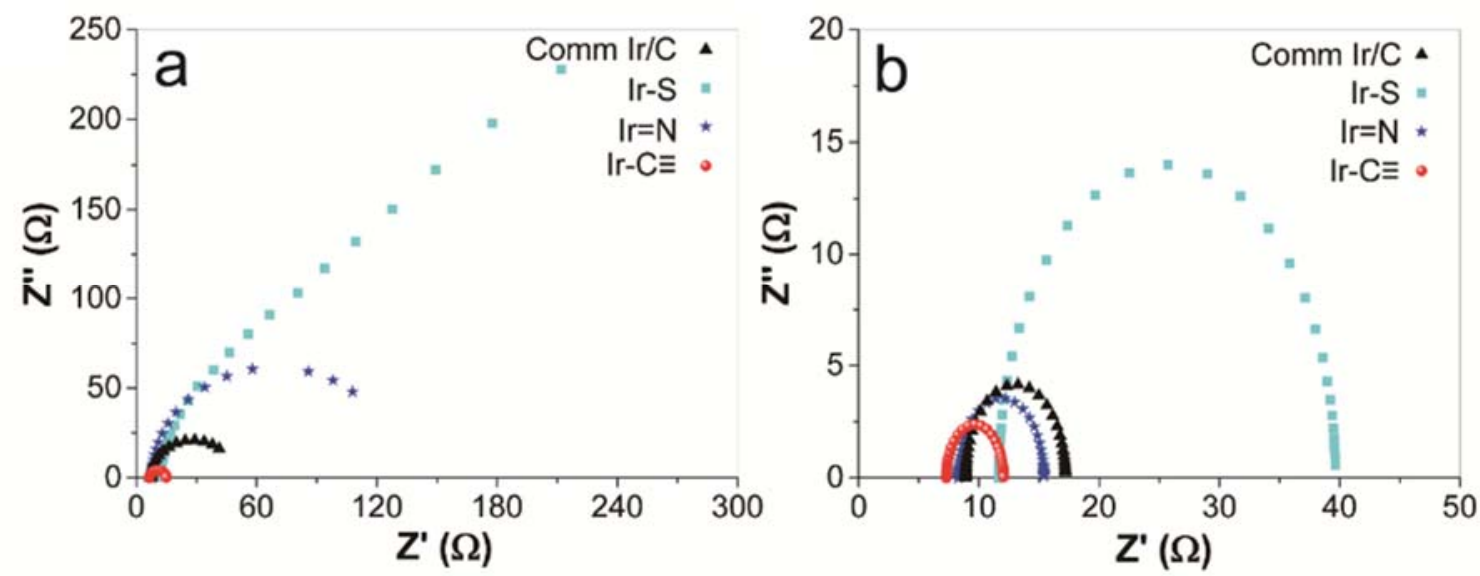

Figure S9. Nyquist plots at the potential of (a) $-100 \mathrm{mV}$ (HER) and (b) $1.50 \mathrm{~V}(\mathrm{OER})$ in $0.5 \mathrm{M} \mathrm{H}_{2} \mathrm{SO}_{4}$.

One can see that the $R_{s}$ increases in the order of $\mathrm{Ir}-\mathrm{C} \equiv<\mathrm{Ir}=\mathrm{N}<\mathrm{Ir}-\mathrm{S}$ (i.e., electrical conductivity increases in the reverse order), consistent with the variation of interfacial charge transfer from the Ir nanoparticle core to the capping ligands.

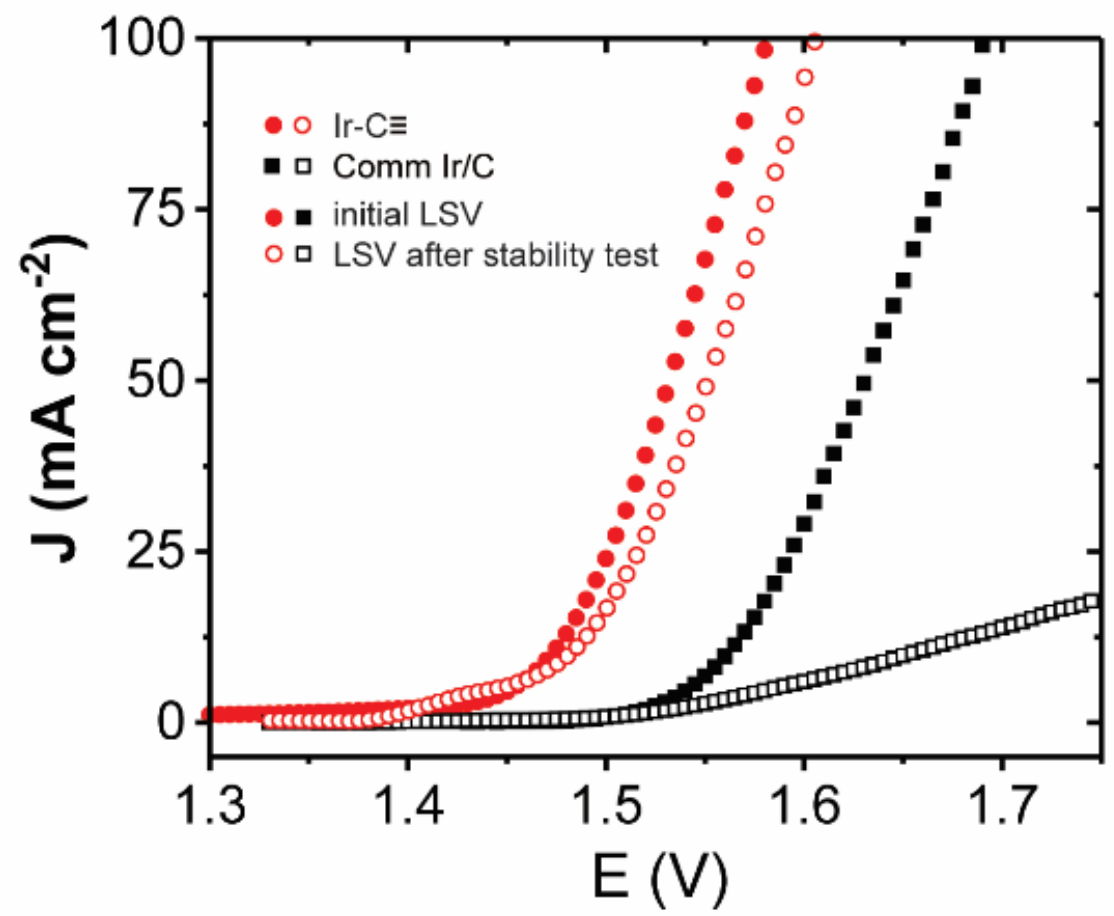

Figure S10. Current-voltage profiles of overall water splitting with $\operatorname{Ir}-\mathrm{C} \equiv$ and commercial $\mathrm{Ir} / \mathrm{C}$ as bifunctional electrocatalysts in $1.0 \mathrm{M} \mathrm{KOH}$ before and after the constant-potential durability test. 

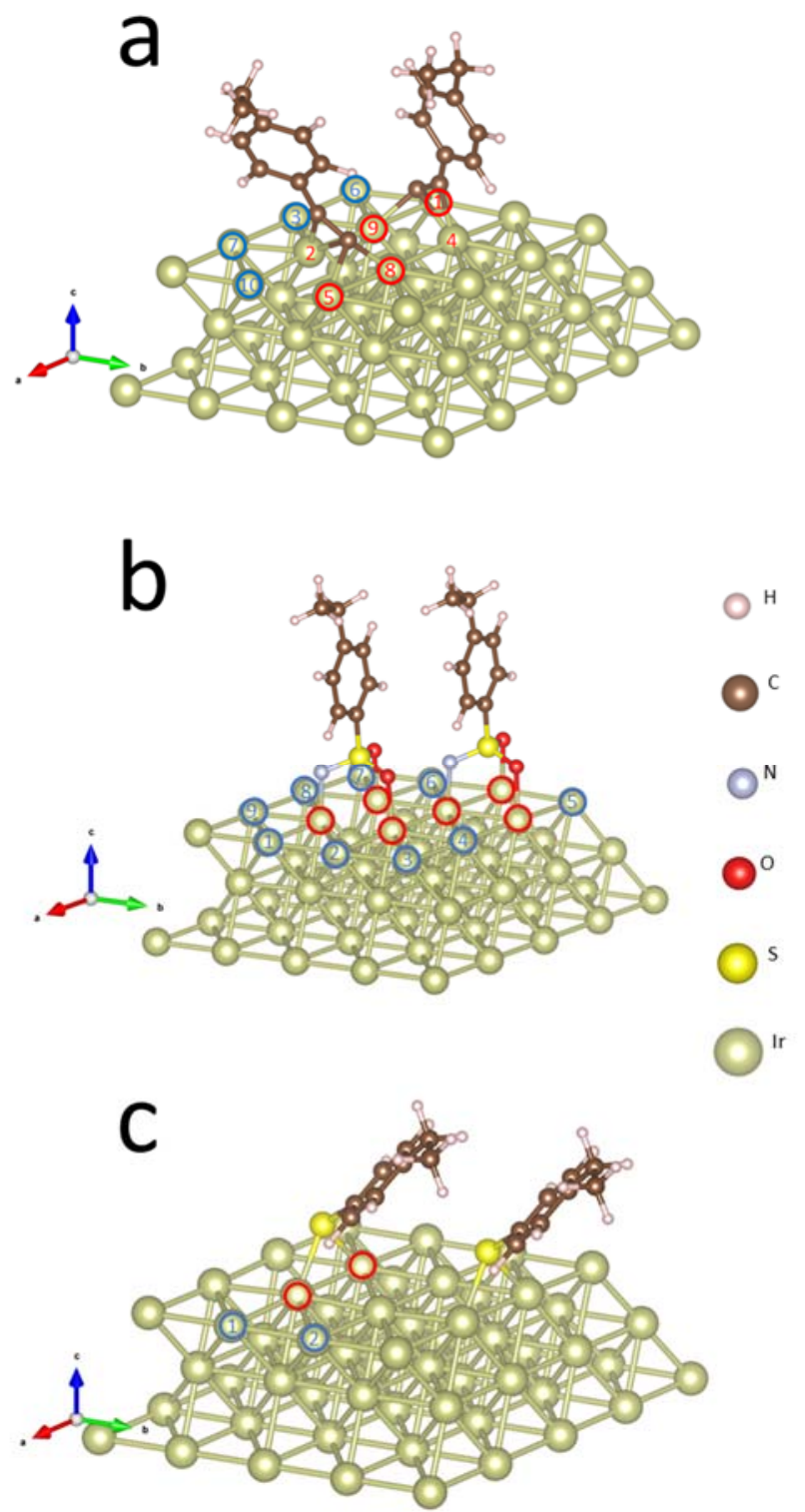

Figure S11. H adsorption sites (ligand-anchoring Ir sites, red circles; neighboring Ir sites, blue circles) in (a) $\mathrm{Ir}-\mathrm{C} \equiv$, (b) Ir=N, and (c) Ir-S. Note that in $\mathrm{H}^{*}$ adsorption calculations, to benchmark the three models, non-monodentate adsorption sites are excluded; and for Ir-S, the sites under the phenyl ring are not considered because of steric effect. The atom numbers correspond to those in the parentheses of Table S2. 

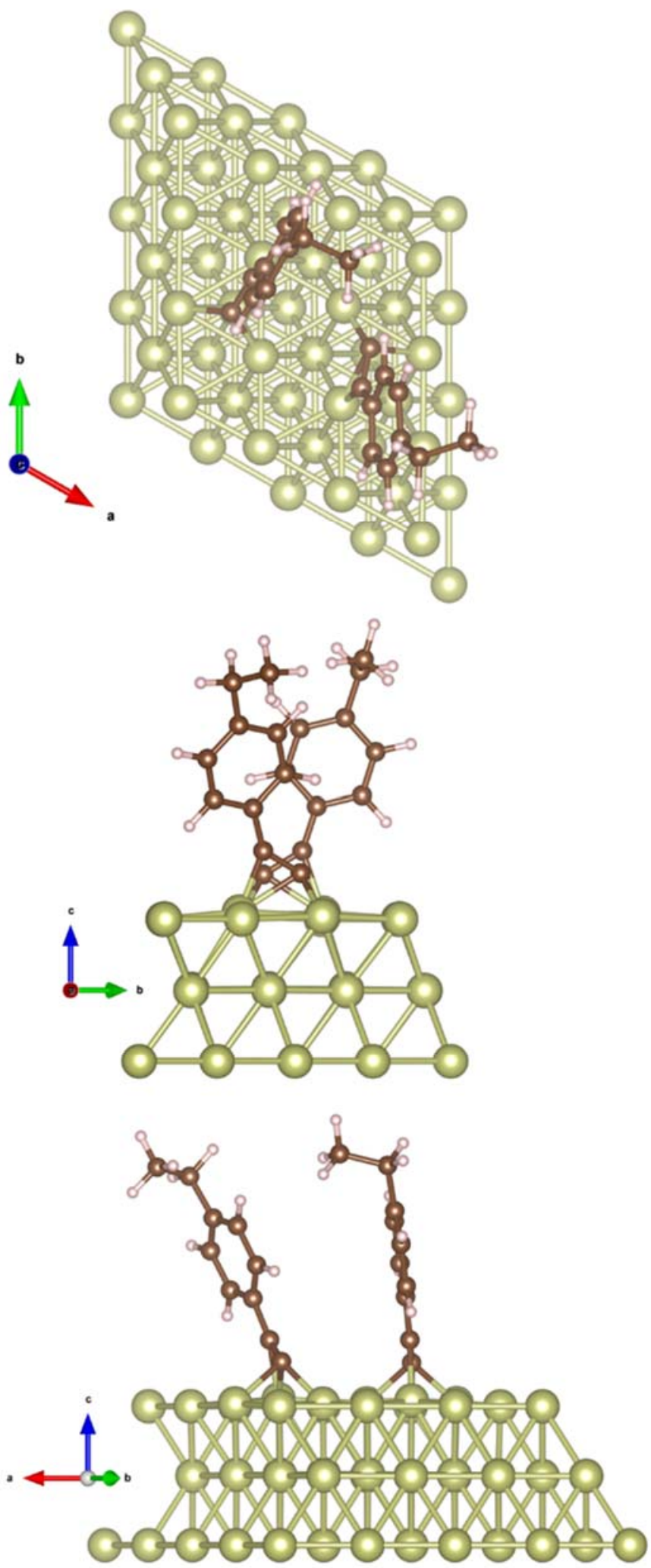

Figure S12. Top and side views of the Ir-C $\equiv$ slab. 


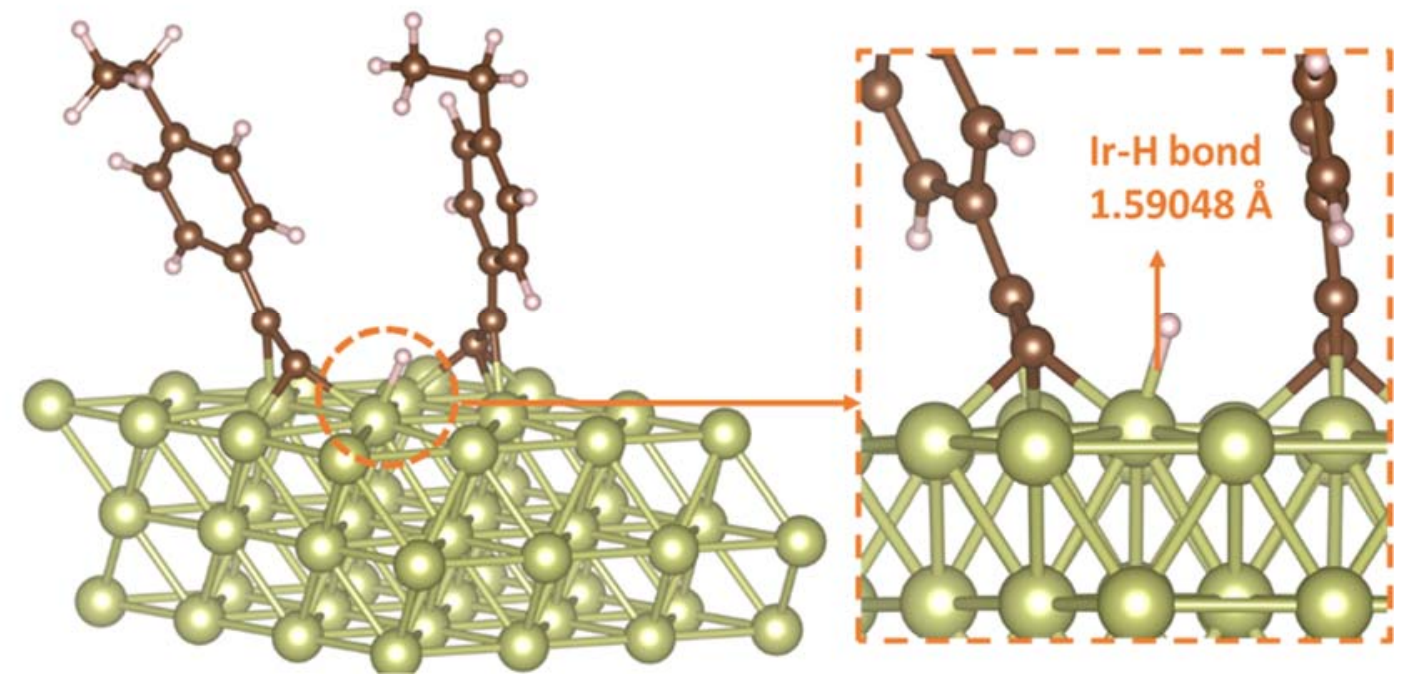

Figure S13. Optimal site in the $\mathrm{Ir}-\mathrm{C} \equiv$ slab for $\mathrm{H}$ adsorption, corresponding to site 8 in Figure $\mathrm{S} 11$. 


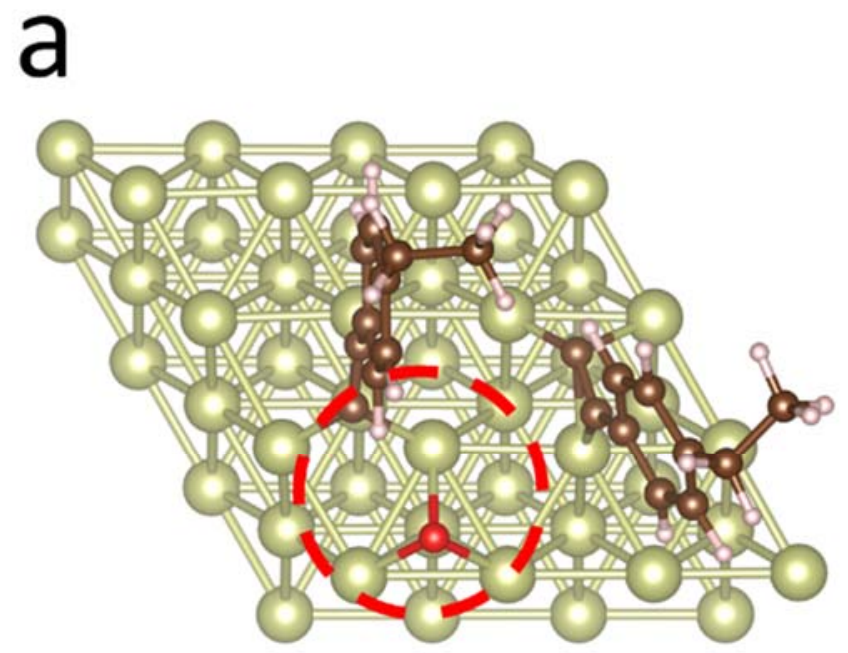

b
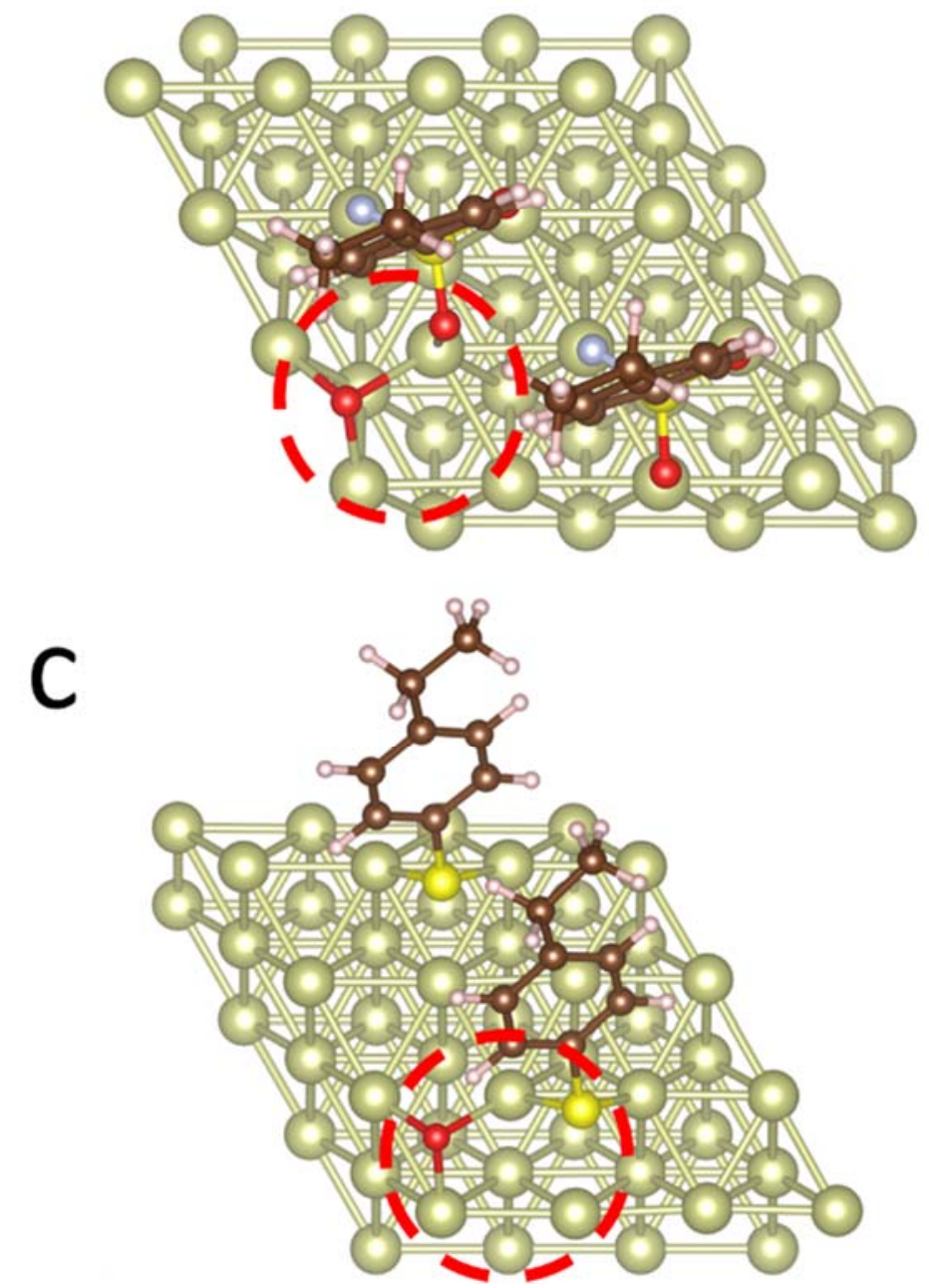

Figure S14. Tridentate adsorption of $\mathrm{O}^{*}$ (circled in red) on (a) $\operatorname{Ir}-\mathrm{C} \equiv,(\mathrm{b}) \operatorname{Ir}=\mathrm{N}$, (c) and Ir-S slabs. 


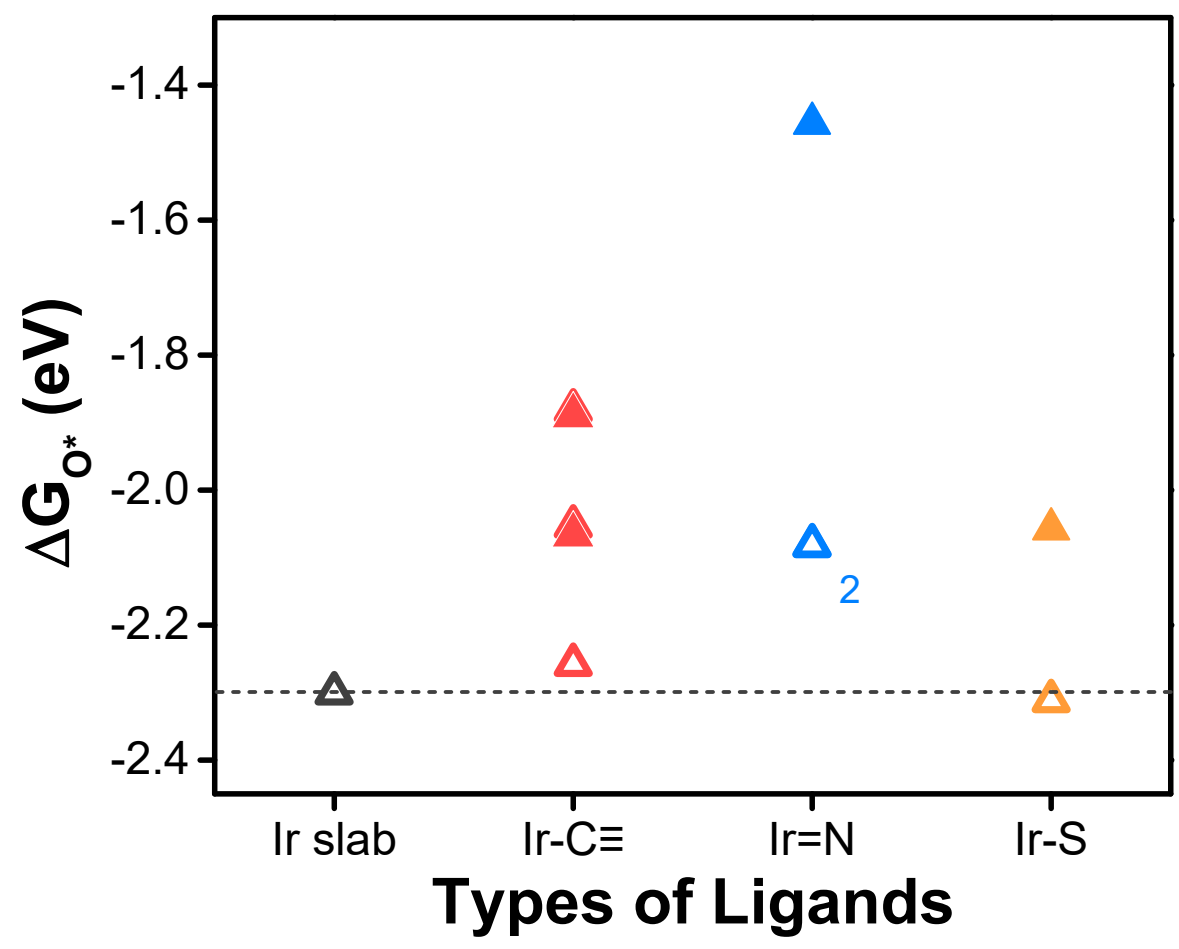

Figure S15 Gibbs free energy of $\mathrm{O}^{*}$ adsorption $\left(\Delta \mathrm{G}^{*}\right)$ for the various models. Note that in $\mathrm{O}^{*}$ adsorption calculation, to benchmark the three models, non-tridentate adsorption sites are excluded; and for Ir-S, the sites under the phenyl ring are not considered because of steric hindrance. 

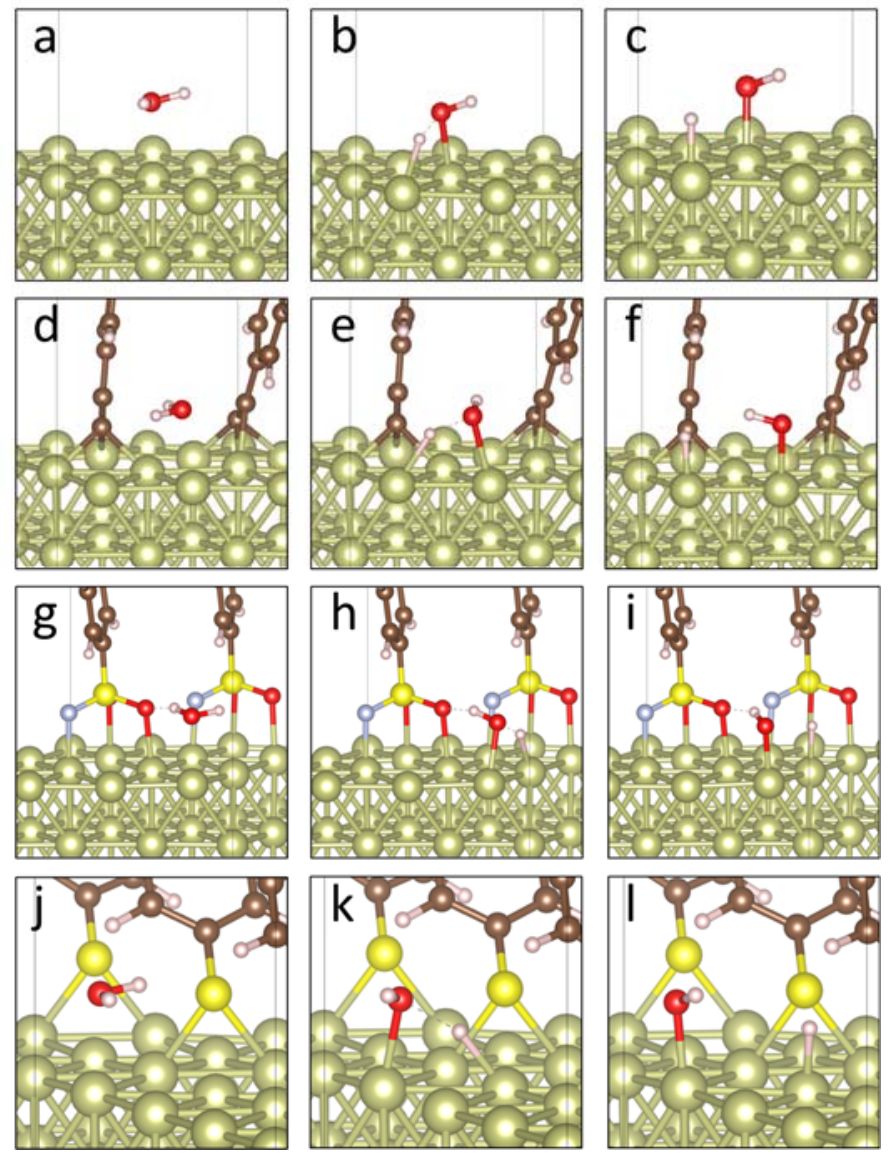

Figure S16. Reaction pathway of water dissociation on (a-c) $\operatorname{Ir}(111),(d-f) \operatorname{Ir}-\mathrm{C} \equiv,(g-i) \operatorname{Ir}=\mathrm{N}$, and (j-I) $\operatorname{Ir}-\mathrm{S}$. Water molecules first approach to $\mathrm{Ir}$ atoms $(\mathrm{a}, \mathrm{d}, \mathrm{g}, \mathrm{j})$ and become dissociated into ${ }^{*} \mathrm{H}$ and ${ }^{*} \mathrm{OH}$ intermediates (b, e, h, k), which are then adsorbed vertically onto two neighboring Ir atoms (c, f, i, I). 
Table S1. Comparison of the HER and OER electrocatalytic performances of the Ir nanoparticles in the present study with relevant catalysts in the literature.

\begin{tabular}{|c|c|c|c|c|c|c|}
\hline catalysts & electrolyte & $\begin{array}{c}\eta_{10, H E R} \\
(\mathrm{mV})\end{array}$ & $\begin{array}{c}\eta_{10, \text { OER }} \\
(\mathrm{mV})\end{array}$ & $E_{10}(V)$ & $\begin{array}{c}\text { Mass } \\
\text { loading } \\
\left(\mu \mathrm{g} \mathrm{cm}^{-2}\right)\end{array}$ & references \\
\hline \multirow{2}{*}{$\operatorname{Ir}-\mathrm{C} \equiv$} & $1.0 \mathrm{M} \mathrm{KOH}$ & -7 & 300 & 1.495 & \multirow{2}{*}{$\sim 40$} & \multirow{2}{*}{ This work } \\
\hline & $0.5 \mathrm{M} \mathrm{H}_{2} \mathrm{SO}_{4}$ & -43 & 256 & 1.473 & & \\
\hline \multirow{2}{*}{ Ir@NG-750 } & $0.5 \mathrm{M} \mathrm{H}_{2} \mathrm{SO}_{4}$ & -19 & 303 & 1.54 & \multirow{2}{*}{11.5} & \multirow{2}{*}{1} \\
\hline & $1.0 \mathrm{M} \mathrm{KOH}$ & -43 & 270 & 1.51 & & \\
\hline \multirow{2}{*}{ Co@Ir/NC } & $0.5 \mathrm{M} \mathrm{H}_{2} \mathrm{SO}_{4}$ & -29.4 & $\mathrm{n} / \mathrm{a}$ & $\mathrm{n} / \mathrm{a}$ & \multirow{2}{*}{357} & \multirow{2}{*}{2} \\
\hline & $1.0 \mathrm{M} \mathrm{KOH}$ & -121 & 280 & 1.631 & & \\
\hline \multirow{3}{*}{ IrNi } & $0.1 \mathrm{M} \mathrm{KOH}$ & -30 & 270 & & \multirow{3}{*}{12.5} & \multirow{3}{*}{3} \\
\hline & $0.1 \mathrm{M} \mathrm{HClO}_{4}$ & -30 & 280 & $\mathrm{n} / \mathrm{a}$ & & \\
\hline & $0.5 \mathrm{M} \mathrm{H}_{2} \mathrm{SO}_{4}$ & $\mathrm{n} / \mathrm{a}$ & $\mathrm{n} / \mathrm{a}$ & 1.58 & & \\
\hline CB(6)-Ir & $0.5 \mathrm{M} \mathrm{H}_{2} \mathrm{SO}_{4}$ & -54 & 270 & 1.56 & 20 & 4 \\
\hline $\begin{array}{c}\text { Culr } \\
\text { nanocages }\end{array}$ & $\begin{array}{l}0.05 \mathrm{M} \\
\mathrm{H}_{2} \mathrm{SO}_{4}\end{array}$ & $\mathrm{n} / \mathrm{a}$ & 286 & $\mathrm{n} / \mathrm{a}$ & $\mathrm{n} / \mathrm{a}$ & 5 \\
\hline $\begin{array}{c}\text { Co-doped } \\
\text { IrCu }\end{array}$ & $0.1 \mathrm{M} \mathrm{HClO}_{4}$ & $\mathrm{n} / \mathrm{a}$ & 293 & $\mathrm{n} / \mathrm{a}$ & 20 & 6 \\
\hline \multirow{3}{*}{ 3D Ir } & $1 \mathrm{M} \mathrm{KOH}$ & \multirow{3}{*}{$\mathrm{n} / \mathrm{a}$} & 245 & \multirow{3}{*}{$\mathrm{n} / \mathrm{a}$} & \multirow{3}{*}{11.5} & \multirow{3}{*}{7} \\
\hline & $0.1 \mathrm{M} \mathrm{KOH}$ & & 296 & & & \\
\hline & $0.1 \mathrm{M} \mathrm{HClO}_{4}$ & & 276 & & & \\
\hline $\mathrm{IrO}_{x} / \mathrm{SrlrO}_{3}$ & $0.5 \mathrm{M} \mathrm{H}_{2} \mathrm{SO}_{4}$ & $\mathrm{n} / \mathrm{a}$ & 290 & $\mathrm{n} / \mathrm{a}$ & $\mathrm{n} / \mathrm{a}$ & 8 \\
\hline Ir/Au & $0.1 \mathrm{M} \mathrm{H}_{2} \mathrm{SO}_{4}$ & $\mathrm{n} / \mathrm{a}$ & 410 & $\mathrm{n} / \mathrm{a}$ & $\mathrm{n} / \mathrm{a}$ & 9 \\
\hline IrNiCu & $0.1 \mathrm{M} \mathrm{HClO}_{4}$ & $\mathrm{n} / \mathrm{a}$ & 300 & $\mathrm{n} / \mathrm{a}$ & 20 & 10 \\
\hline IrCo NPs & $0.5 \mathrm{M} \mathrm{H}_{2} \mathrm{SO}_{4}$ & -24 & 270 & 1.55 & 18.9 & 11 \\
\hline \multirow{3}{*}{ IrW } & $0.1 \mathrm{M} \mathrm{H}_{2} \mathrm{SO}_{4}$ & -16 & 271 & 1.58 & \multirow{3}{*}{60} & \multirow{3}{*}{12} \\
\hline & $0.1 \mathrm{M} \mathrm{KOH}$ & -29 & 281 & 1.6 & & \\
\hline & $1 \mathrm{M}$ PBS & -35 & 326 & 1.73 & & \\
\hline \multirow{2}{*}{ AuCu@IrNi } & $0.5 \mathrm{M} \mathrm{H}_{2} \mathrm{SO}_{4}$ & $\mathrm{n} / \mathrm{a}$ & $\mathrm{n} / \mathrm{a}$ & 1.585 & \multirow{2}{*}{100} & \multirow{2}{*}{13} \\
\hline & $0.1 \mathrm{M} \mathrm{HClO}_{4}$ & -12.7 & 308 & $\mathrm{n} / \mathrm{a}$ & & \\
\hline \multirow{3}{*}{ 3D IrNiCo } & $0.1 \mathrm{M} \mathrm{HClO}_{4}$ & $\mathrm{n} / \mathrm{a}$ & 220 & $\mathrm{n} / \mathrm{a}$ & \multirow{3}{*}{250} & \multirow{3}{*}{14} \\
\hline & $0.1 \mathrm{M} \mathrm{KOH}$ & $\mathrm{n} / \mathrm{a}$ & 190 & $\mathrm{n} / \mathrm{a}$ & & \\
\hline & PBS & $\mathrm{n} / \mathrm{a}$ & 290 & $\mathrm{n} / \mathrm{a}$ & & \\
\hline \multirow{2}{*}{$\mathrm{IrP}_{2} @ \mathrm{NC}$} & $0.5 \mathrm{M} \mathrm{H}_{2} \mathrm{SO}_{4}$ & -8 & $\mathrm{n} / \mathrm{a}$ & $\mathrm{n} / \mathrm{a}$ & \multirow{2}{*}{$\mathrm{n} / \mathrm{a}$} & \multirow{2}{*}{15} \\
\hline & $1.0 \mathrm{M} \mathrm{KOH}$ & -28 & $\mathrm{n} / \mathrm{a}$ & $\mathrm{n} / \mathrm{a}$ & & \\
\hline IrP & $0.1 \mathrm{M} \mathrm{HClO}_{4}$ & & -260 & & 230 & 16 \\
\hline $\mathrm{Ir} / \mathrm{GF}$ & $0.5 \mathrm{M} \mathrm{H}_{2} \mathrm{SO}_{4}$ & -7 & 290 & 1.55 & & 17 \\
\hline IrNSs & $0.5 \mathrm{M} \mathrm{H}_{2} \mathrm{SO}_{4}$ & $\mathrm{n} / \mathrm{a}$ & 240 & $\mathrm{n} / \mathrm{a}$ & 137 & 18 \\
\hline $\mathrm{IrO}_{2}$ & $0.5 \mathrm{M} \mathrm{H}_{2} \mathrm{SO}_{4}$ & $\mathrm{n} / \mathrm{a}$ & 313 & $\mathrm{n} / \mathrm{a}$ & 250 & 19 \\
\hline
\end{tabular}


Table S2. Gibbs free energy of $\mathrm{H}^{*}$ adsorption $\left(\Delta \mathrm{G}_{\mathrm{H}^{*}}\right)$ on bare $\operatorname{Ir}(111)$ slab and on ligand-anchoring Ir atoms (blue data points) and their neighboring Ir atoms in different organically capped slabs (the numbers in the red parentheses are the corresponding atoms shown in Figure S11).

\begin{tabular}{|c|c|c|c|c|c|c|}
\hline & \multicolumn{5}{|c|}{$\Delta \mathrm{G}_{\mathrm{H}^{*}}(\mathrm{eV})$} & average $\Delta \mathrm{G}_{\mathrm{H}^{*}}(\mathrm{eV})$ \\
\hline bare slab & \multicolumn{5}{|c|}{-0.31989} & -0.31989 \\
\hline \multirow{2}{*}{ Ir-C $\equiv$} & $-0.34515(10)$ & $-0.18519(7)$ & $-0.30398(3)$ & $-0.28365(6)$ & & $-0.27949 \pm 0.05878$ \\
\cline { 2 - 7 } & $-0.08569(9)$ & $-0.07487(5)$ & $-0.11278(1)$ & $0.01513(8)$ & & $-0.06455 \pm 0.04803$ \\
\hline \multirow{2}{*}{ Ir=N } & $-0.26059(5)$ & $-0.28896(6)$ & $-0.29722(7)$ & $-0.2753(8)$ & $-0.28383(9)$ & $-0.28404 \pm 0.01093$ \\
\cline { 2 - 7 } & $-0.28392(1)$ & $-0.27886(2)$ & $-0.2978(3)$ & $-0.28989(4)$ & & $-0.31939 \pm 0.00773$ \\
\hline \multirow{2}{*}{ Ir-S } & $-0.31166(1)$ & $-0.32711(2)$ & & & & - \\
\hline
\end{tabular}

\#

Table S3. Bader charge calculations of ligand-anchoring Ir sites (blue data) and neighboring Ir sites (black data) on Ir-CE, as specified in Figure S11.

\begin{tabular}{cccc}
\hline Ir atom number & $\begin{array}{c}\text { Charge with ligands } \\
(\mathrm{e})\end{array}$ & $\begin{array}{c}\text { Charge without ligand } \\
(\mathrm{e})\end{array}$ & $\begin{array}{c}\text { Charge transfer } \\
(\mathrm{e})\end{array}$ \\
\hline 1 & 14.89466 & 15.0411 & -0.14644 \\
2 & 14.85723 & 15.04027 & -0.18304 \\
3 & 15.05838 & 15.04811 & 0.010272 \\
4 & 14.85255 & 15.0555 & -0.20296 \\
5 & 14.8955 & 15.05135 & -0.15585 \\
6 & 15.01694 & 15.02026 & -0.00332 \\
7 & 15.08258 & 15.04198 & 0.040595 \\
8 & 14.87658 & 15.05934 & -0.18276 \\
9 & 14.92141 & 15.07618 & -0.15477 \\
10 & 15.05699 & 15.05161 & 0.005381 \\
\hline
\end{tabular}




\section{References}

1. Wu, X. J.; Feng, B. M.; Li, W.; Niu, Y. L.; Yu, Y. A.; Lu, S. Y.; Zhong, C. Y.; Liu, P. Y.; Tian, Z. Q.; Chen, L.; Hu, W. H.; Li, C. M., Metal-support interaction boosted electrocatalysis of ultrasmall iridium nanoparticles supported on nitrogen doped graphene for highly efficient water electrolysis in acidic and alkaline media. Nano Energy 2019, 62, 117-126.

2. Li, D. L.; Zong, Z.; Tang, Z. H.; Liu, Z.; Chen, S. W.; Tian, Y.; Wang, X. F., Total Water Splitting Catalyzed by Co@Ir Core-Shell Nanoparticles Encapsulated in Nitrogen-Doped Porous Carbon Derived from Metal Organic Frameworks. ACS Sustain Chem Eng 2018, 6 (4), 5105-5114.

3. Pi, Y. C.; Shao, Q.; Wang, P. T.; Guo, J.; Huang, X. Q., General Formation of Monodisperse IrM (M = Ni, $\mathrm{Co}, \mathrm{Fe})$ Bimetallic Nanoclusters as Bifunctional Electrocatalysts for Acidic Overall Water Splitting. Adv Funct Mater 2017, 27 (27), 1700886.

4. You, H. H.; Wu, D. S.; Chen, Z. N.; Sun, F. F.; Zhang, H.; Chen, Z. H.; Cao, M. N.; Zhuang, W.; Cao, R., Highly Active and Stable Water Splitting in Acidic Media Using a Bifunctional Iridium/Cucurbit[6]uril Catalyst. ACS Energy Lett 2019, 4 (6), 1301-1307.

5. Wang, C.; Sui, Y. M.; Xiao, G. J.; Yang, X. Y.; Wei, Y. J.; Zou, G. T.; Zou, B., Synthesis of Cu-Ir nanocages with enhanced electrocatalytic activity for the oxygen evolution reaction. J Mater Chem $A$ 2015, 3 (39), 19669-19673.

6. Kwon, T.; Hwang, H.; Sa, Y. J.; Park, J.; Baik, H.; Joo, S. H.; Lee, K., Cobalt Assisted Synthesis of IrCu Hollow Octahedral Nanocages as Highly Active Electrocatalysts toward Oxygen Evolution Reaction. Adv Funct Mater 2017, 27 (7), 1604688.

7. Pi, Y. C.; Zhang, N.; Guo, S. J.; Guo, J.; Huang, X. Q., Ultrathin Laminar Ir Superstructure as Highly Efficient Oxygen Evolution Electrocatalyst in Broad pH Range. Nano Lett 2016, 16 (7), 4424-4430.

8. Seitz, L. C.; Dickens, C. F.; Nishio, K.; Hikita, Y.; Montoya, J.; Doyle, A.; Kirk, C.; Vojvodic, A.; Hwang, H. Y.; Norskov, J. K.; Jaramillo, T. F., A highly active and stable $1 \mathrm{rO}_{x} / \mathrm{SrlrO}_{3}$ catalyst for the oxygen evolution reaction. Science 2016, 353 (6303), 1011-1014.

9. Ahn, S. H.; Tan, H. Y.; Haensch, M.; Liu, Y. H.; Bendersky, L. A.; Moffat, T. P., Self-terminated electrodeposition of iridium electrocatalysts. Energ Environ Sci 2015, 8 (12), 3557-3562.

10. Park, J.; Sa, Y. J.; Baik, H.; Kwon, T.; Joo, S. H.; Lee, K., Iridium-Based Multimetallic Nanoframe@Nanoframe Structure: An Efficient and Robust Electrocatalyst toward Oxygen Evolution Reaction. ACS Nano 2017, 11 (6), 5500-5509.

11. Sun, X. C.; Liu, F.; Chen, X.; Li, C. C.; Yu, J.; Pan, M., Iridium-doped ZIFs-derived porous carbon-coated IrCo alloy as competent bifunctional catalyst for overall water splitting in acid medium. Electrochim Acta 2019, 307, 206-213.

12. Fu, L. H.; Hu, X.; Li, Y. B.; Cheng, G. Z.; Luo, W., IrW nanobranches as an advanced electrocatalyst for pH-universal overall water splitting. Nanoscale 2019, 11 (18), 8898-8905.

13. Park, J.; Choi, S.; Oh, A.; Jin, H.; Joo, J.; Baik, H.; Lee, K., Hemi-core@frame AuCu@IrNi nanocrystals as active and durable bifunctional catalysts for the water splitting reaction in acidic media. Nanoscale Horiz 2019, 4 (3), 727-734.

14. Zhao, Y.; Luo, M.; Chu, S. F.; Peng, M.; Liu, B. Y.; Wu, Q. L.; Liu, P.; de Groot, F. M. F.; Tan, Y. W., 3D nanoporous iridium-based alloy microwires for efficient oxygen evolution in acidic media. Nano Energy 2019, 59, 146-153. 
15. Pu, Z. H.; Zhao, J. H.; Amiinu, I. S.; Li, W. Q.; Wang, M.; He, D. P.; Mu, S. C., A universal synthesis strategy for P-rich noble metal diphosphide-based electrocatalysts for the hydrogen evolution reaction. Energy Environ Sci 2019, 12 (3), 952-957.

16. Yin, L. S.; Yang, T.; Ding, X. R.; He, M. S.; Wei, W. X.; Yua, T. T.; Zhaoa, H., Synthesis of phosphorus-iridium nanocrystals and their superior electrocatalytic activity for oxygen evolution reaction. Electrochem Commun 2018, 94, 59-63.

17. Zhang, J.; Wang, G.; Liao, Z. Q.; Zhang, P. P.; Wang, F. X.; Zhuang, X. D.; Zschech, E.; Feng, X. L., Iridium nanoparticles anchored on 3D graphite foam as a bifunctional electrocatalyst for excellent overall water splitting in acidic solution. Nano Energy 2017, 40, 27-33.

18. Jiang, B.; Guo, Y. N.; Kim, J.; Whitten, A. E.; Wood, K.; Kani, K.; Rowan, A. E.; Henzie, J.; Yamauchi, Y., Mesoporous Metallic Iridium Nanosheets. J Am Chem Soc 2018, 140 (39), 12434-12441.

19. Lim, J.; Park, D.; Jeon, S. S.; Roh, C. W.; Choi, J.; Yoon, D.; Park, M.; Jung, H.; Lee, H., Ultrathin IrO2 Nanoneedles for Electrochemical Water Oxidation. Adv Funct Mater 2018, 28 (4), 1704796. 